16

\section{RELATIVE POVERTY AND CHILD MORTALITY IN HAITI}

A Hill, B Shaffer, E Doucette, S Obenhaus, S Shaffer, E. Grey Dimond MD International Medicine Program, The University of Missouri - Kansas City, School of Medicine, Kansas City, MO

Haiti suffers from both the worst poverty and highest child mortality rates in the Western Hemisphere. The purpose of this investigation was to assess the relationship of child mortality to relative degrees of poverty within Haiti. Method: We conducted a survey of 656 women, 15-45 year of age, living in a particularly poor rural area of southern Haiti. Data was collected regarding living conditions (dwelling size, occupants, construction characteristics, presence of a latrine, and water access) and pregnancy outcome (number of pregnancies, pregnancy losses, stillbirths, and child deaths). Relative poverty, as indicated by living conditions, was compared with pregnancy outcome using pooled and individual variables.

Results: Using a scoring system for pooled variables, a significant correlation was found between overall living conditions and overall pregnancy outcome, i.e. women living in a thatched house wit a dirt floor and no latrine were more likely to have a pregnancy loss, stillbirth, or child death when compared to mothers living in a house with a cement floor, sheet metal roof, and access to a latrine $(\mathrm{p}<0.01)$. Among individual descriptors of poverty, we noted that women living in a dwelling with a dwellings with a cement floor reported 63 child deaths following 1101 live births $(6 \%), p=0.01$, relative risk of child mortality $=1.5$

Conclusion: Child mortality risk is heterogeneous. Even within a poor neighborhood in a poor country, stratifications of poverty and health outcome are discernable. Certain living conditions may be practical indicators of risk and thereby useful to direct interventions and resources.

\section{7}

TRADITIONAL GROWTH CURVE COMPARED WITH CONTEMPORARY LOCAL CURVE FAILS TO IDENTIFY SGA INFANTS.

Kerri Fitzgerald, M.D., Gary Pettett, M.D., Felix A. Okah, M.D., Stephen D. Simon Ph.D., Deborah Biondo, MS,RD,LD,CNSD, Howard W. Kilbride, M.D. Department of Pediatrics, Children's Mercy Hospitals and Clinics, Kansas City MO, United States

Background: The classification of small for gestation age (SGA) is defined as birth weight below the $10^{\text {th }}$ percentile on growth charts. A commonly used growth chart, Lubchenco (Pediatrics, 1969), was developed in the1960's, but concerns have been raised about its validity due to its use of population at high altitude and the exclusion of racial/ethnic minorities. SGA is associated with infant morbidity and mortality and may be associated with adult-onset disease, the "metabolic syndrome." The utilization of the traditional growth curve may underestimate the number of children who are born SGA and who have additional neonatal morbidities and increased risk of life-long chronic disease.

Objective: To compare the $10^{\text {th }}$ percentile birth weights in a Kansas City newborn sample using the Lubchenco growth curve compared to a curve generated by a local population data.

Design/Methods: Retrospective cohort study of births at Truman Medical Center in Kansas City, Missouri. The birth records of 5,687 sequential births were assessed to obtain gestational age based o maternal data (last menstrual period or prenatal ultrasound) and birth measurements. Outcome of interest was SGA. The $10^{\text {th }}, 50^{\text {th }}$ and $90^{\text {th }}$ percentiles for each gestational age were calculated. Deviations from the Lubchecno growth curves were calculated for each gestational age. The proportion Deviations from the Lubchecno growth curves were calculated for each gestational age. The p.
of SGA in the Kansas City cohort, as defined by the Lubchenco chart, was also computed.

Results: For infants with gestational age of 32 weeks and above, the Kansas City cohort had substantially larger values for the $10^{\text {th }}$ percentile. The average deviation was $9 \%$ (range: $4 \%$ to $16 \%$ ) The proportion of the Kansas City cohort that would be classified as SGA by the Lubchenco growt curve would only be $5 \%$, less than half of the expected value (95\% confidence interval $4.5 \%$ to $5.6 \%$ ).

Conclusion: A significant number of infants born after 32 weeks gestation are SGA when plotted on locally-generated growth curves compared to the Lubchenco growth curves. It is apparent that the commonly used Lubchenco growth curves may not be appropriate for recognizing SGA in our population. Future investigations will determine whether or not the newborns whose diagnosis has been missed by the use of the Lubchenco charts suffer the morbidity and mortality associated with SGA.

\section{8}

\section{IDENTIFICATION OF A NOVEL NONSENSE MUTATION IN THE EYA1 GENE ASSOCIATED WITH BRANCHIOOTIC/BRANCHIO-OTO-RENAI SYNDROME.}

J. Clarke* E. M. Honey\$, R. M. Raymond Jr.*, C. Lord* and P. D. Brophy*, \$ University of Pretoria Department of Genetics, Pretoria, South Africa,*University of Michigan Department of Pediatrics, Ann Arbor, MI.

The most frequent causes of chronic renal failure in the first two decades of life are due to urinary tract . mental disorder of the kidney and urinary tract, accompanied with hearing loss and presenting with a wide intrafanilial variability and reduced penetrance. Dominant mutations in the human homologues of the Drosophila eyes absent gene (EYA1) are frequently the cause of BOR and BO syndromes. EYA1 encodes a 559-amino acid polypeptide with a predicted molecular mass of 61.2

novel nonsense mutation in EYA1 as the cause of BOR in this family.
Family JCA7 is of South African Caucasian descent and was identified during routine prenatal ultrasound Family JCA7 is of South African Caucasian descent and was identified during routine prenatal ultrasoun screening. The proband (II.2) was noted to have absent kidneys and oligohydramnios during this initia screening. A review of the health histories of the family indicated a history of Potter Sequence in a previous pregnancy (II.3) and that the father (1.1) has a unilateral pre-auricular pit with ipsilateral hearing los. Preliminary renal ultrasound scans of I.1, the mother (1.2) and unaffected daughter (II.1) did not reveal an abnormalitics of the uiogenital systich dysplastic kidney was present. The infant also presented with a hypoplastic bladder, Potter facies and pulmonary hypoplasia. Bas of the EYA1 gene for mutationa analysis. (University of Michigan IRB \# 2004-0322). Sequence analysis revealed that the patients I.1 and II.2 caried a hetezidion of this mutation results in E243X that presumably truncates the full length EYA1 protein by the introduction of a stop codon. The E243X mut pren in the affected members of this family should result in a protein product lacking the critical EYA homologous region (EYAhr) encoded by exons 9 through 16. The EYAh is imperative for the ability of the EYAl protein product to bind to other proteins, such as SIX1, and regulate transcription properly. Functional analysis is currently being performed in order to assess the mutational
effect on binding of protein complex partners and DNA target sequences. Phenotype/genotype correlations effect on binding of protein complex partners and DNA target sequences. Phenotyp
are also being investigated as more samples from the kindred become available.

\section{9}

CLINICIAN'S APPROACH TO PERTUSSIS DURING AN OUTBREAK YEAR. AL Myers, M A Jackson, S Simon, Children's Mercy Hospitals and Clinics, Kansas City, MO, University of Missouri at Kansas City.

BACKGROUND: DTaP was approved for use in the primary vaccine series for US children in 1996. Despite vaccine coverage rates estimated at $85 \%$, as many as 10,000 cases of pertussis are reported annually in the US. In 2004, 79 children in Kansas City, MO had confirmed pertussis infection representing a 3 fold increase compared to 20 prior yrs, and doubling of the prior $4 \mathrm{yrs}$.

METHODS: Following IRB approval, all cases of pertussis reported by our infection control department were identified. Clinical and epidemiologic data was collected from visit records for infants and children diagnosed with pertussis $1 / 1 / 00-12 / 31 / 03$ and was compared to data from 1/1/04-12/ $31 / 04$

RESULTS: The diagnosis of pertussis was confirmed in 79 children in 2004, compared to an average of 30/yr in 2000-03, and 20/yr in 1984-99. The \% of children less than 6 mos was simila overall. There was an increase in children $>10 \mathrm{yrs}$ in the 2004 cohort $(\mathrm{p}<0.0001)$. Hispanic children represented $15 \%$ of cases in 2004 (locally $7 \%$ of pop), twice as many as the previous 4 yrs combined. The percentage of children receiving state supported health coverage increased from $50 \%$ to $68 \%$ ( $p=$ $0.013)$. More children were seen in the urgent care and ED setting $(\mathrm{p}=0.076)$.

Typical presentations were recorded for infected children, though omissions in the clinical description were common. $29 \%$ of the time, pertussis was not suspected at initial presentation. In those who received pertussis testing, correct treatment was prescribed in $62 \%$ of cases. Vaccine status was not recorded in $48 \%$ of patients $>2$ mos. Contact isolation was discussed in $8 \%$ of cases, and household chemoprophylaxis was provided in $51 \%$.

CONCLUSION: Despite good vaccine coverage rates in the US, pertussis continues to be reported; 2004 represented the largest outbreak in the last $30 \mathrm{yrs}$. Disease was under diagnosed, vaccine status was often not recorded, and household prophylaxis delayed in most cases. The addition of vaccine for adolescents will have some impact, but strategies to improve control should also include increasing $\mathrm{DTaP}$ coverage rate for infants, consistent use of a clinical definition to diagnose pertussis, prompt treatment of the index case, and prophylaxis of household contacts.

\section{0}

\section{USE OF NONINVASIVE POSITIVE PRESSURE VENTILATION TO FACILI- TATE EXTUBATION IN PEDIATRIC PATIENTS WITH NEUROMUSCULAR DISEASES.}

TA Shah, GR Paul, DJ Birnkrant, Department of Pediatrics, MetroHealth Medical Center, Case Western Reserve University School of Medicine, Cleveland, OH.

Introduction: Patients with neuromuscular diseases (NMDs) who require intubation are at risk of extubation failure due to respiratory muscle weakness and they may require tracheostomy. Purpose: To evaluate the use of noninvasive positive pressure ventilation (NPPV) with the BiPAP® (Respironics) device to facilitate extubation of children with NMDs and poor baseline pulmonary function who have been intubated for acute respiratory failure or surgery. Methods: Retrospective chart review, IRB approved. Results: During the time period Jan 2001-Dec 2004, NPPV was used to facilitate extubation for 22 episodes of intubation in 14 patients. Diagnoses: Duchenne muscular dystrophy (Duchenne MD) (7 patients); type 1 spinal muscular atrophy (2 patients); Becker MD (1 patient); congenital MD (1 patient); nemaline myopathy (1 patient); polymyositis (1 patient); acid maltase deficiency (1 patient). Mean age at time of extubation ( $\pm \mathrm{SD}$ ): $10.7 \pm 5.9$ years (range: $1-20$ years); 11 of 14 patients were male. Four of 22 episode of extubation were after spinal surgery. In these patients, mean baseline vital capacity was $47.3 \pm 11.4 \%$ predicted. In 18 episodes, extubation was after acute respiratory illness with respiratory failure. Mean baseline vital capacity for these patients: $24.5 \pm 9.1 \%$ predicted (range: $11-38 \%)(\mathrm{n}=10$, excludes spinal muscular atrophy infants). NPPV was used successfully to facilitate extubation in all 4 episodes of post-surgical extubaton. NPPV was successful in 17 of 18 episodes of extubation during acute respiratory illnesses (94\% success rate). At follow-up (mean $27.5 \pm 15.2$ months after extubation), 2 patients have died (1 patient had Duchenne MD and refused chronic NPPV, 1 patient had acid maltase deficiency). The remaining 12 patients are alive, 9 of whom have no tracheostomy and are using chronic home NPPV. The mean vital capacity at follow-up in these patients is $27.5 \pm 14.2 \%$ predicted. Two patients are now ventilated with tracheostomy tubes. Conclusion: Patients with severe neuromuscular diseases who have been intubated are at risk for extubation failure and the need for tracheostomy. Our study suggests that, despite highly impaired pulmonary function, NPPV can be used to extubate many of these patients successfully. On long-term follow-up, most of our patients have avoided tracheostomy. Further study is needed to optimize the use of NPPV for patients with NMDs.

\section{1}

PROGNOSTIC FACTORS IN CHILDHOOD NONRHABDOMYOSARCOMA SOFT TISSUE SARCOMAS

Ram Kalpatthi, MD, Frederick B Ruymann, MD, Mohamed Bayoumy, MD, Kathryn J Klopfenstein, MD, Jamil Altaf, MD, Division of Pediatric Hematology/Oncology, Children's Hospital, Columbus, OH

Background: Non-rhabdomyosarcoma soft tissue sarcomas (NRSTS) represent about $3 \%$ of all childhood malignancies and about $50 \%$ of all soft tissue sarcomas. The heterogeneity of these tumors accounts for the paucity of information regarding their natural history and treatment guidelines. Therefore much of the evidence regarding the therapy has been extrapolated from adult studies and few pediatric case series. The purpose of this study is to evaluate our clinical experience with NRSTS in the pediatric population over the past 50 years.

Objectives: To evaluate the prognostic variables, event free survival and overall survival of childhood NRSTS.

Methods: We reviewed the medical records of all patients less than 21 years of age with NRSTS treated at our hospital between 1945 and 2001. Patients with Ewing's family of soft tissue sarcomas were excluded from the analysis. We collected the demographic, disease, histopathology and treatment data of 80 patients in whom all the data were available. The patient's age ranged from 1 month to 20 data of 80 patients in whom all the data were available. The patient's age ranged from 1 month to 20
years (median 11.5 years). The median follow up time was 6 years ( 1 month to 46 years). Event free survival (EFS) and overall survival (OS) was calculated using Kaplan-Meier survival method.

Results: Of the 80 patients, $34(42.5 \%)$ were able to achieve a complete surgical resection. Forty four $(55 \%)$ received external beam radiotherapy and adjuvant chemotherapy was given to $57(71.3 \%)$ cases. The EFS was significantly higher for patients with Stage I/II tumors (73\%) as compared to Stage III/IV tumors (23\%) [EFS, log rank $=18.8, \mathrm{p}<0.001 ; \mathrm{OS}, \log$ rank $=21.9, \mathrm{p}<0.001]$. Of the different treatment modalities only complete surgical resection had a significant impact on survival (log rank $=$ $8.9, \mathrm{p}<0.003$ ). Relative to other sites, chest and abdominal tumors had advanced stages (Chi Square $=$ $22.9, p=0.029$ ). None of the other variables such as age, sex or race impacted survival in our study. Conclusions: Our data show that surgical resection and tumor stage are the most importan prognostic variables in childhood NRSTS. Wide surgical resection of the primary tumor with negative margins continues to be the mainstay of therapy. Radiotherapy and adjuvant chemotherapy should be reserved for patients with inadequate margins or those in whom a complete surgical resection can be facilitated by these modalities. 